\title{
Targeted therapy in Head and Neck Cancer: Some Success But More Is Expected!
}

Squamous cell carcinoma of head and cancer (SCCHN) constitutes about $21 \%$ of all cancer in this country, a significant number. Unfortunately, majority present with advanced stage or recur after initial definitive therapy. Despite significant advances in surgical, radiation and medical treatment, outcome and prognosis of recurrent SCCHN is poor. One field which is contributing significantly in the development of new drugs, is molecular biology. Better understanding of cancer pathogenesis, pathways involved, growth (inhibitory or stimulatory) factors, and knowledge of proteins involved in these activities have led to the concept of targeted therapy. Theoretically, these agents act on specific cellular pathways and receptor expressed on cancer cells and thereby are devoid of usual toxicity seen with cytotoxic chemotherapy. Ever since the success of lmatinib for chronic myeloid leukemia (CML) rituximab (anti CD 20 antibody) for non Hodgkin's Lymphoma and transtuzumab (herceptin) for breast cancer there has been spurt in research for development of new-targeted agents for solid and haematological cancers. Head and neck cancer which is known to express epidermal growth factor receptors (EGFR) also has been the focus for targeted therapy. Agents being studied are either monoclonal antibodies (cetuximab, panitumumab, matuzumab) or tyrosine kinase inhibitor (gefitinib or erlotinib etc.). Monoclonal antibody against EGFR, cetuximab has been found useful along with radiation therapy (RT) as first line therapy compared to RT alone, ${ }^{1}$ and along with platinum based chemotherapy in recurrent cancer. ${ }^{2}$ However, the true value of its usefulness will be seen only after results from ongoing studies are available. In this issue of IJMPO, Rao et al have reported their experience with gefitinib (a tyrosine kinase inhibitor) in the treatment of recurrent SCCHN. ${ }^{3}$ Authors have reported symptomatic improvement in about $63 \%$ of patients and radiological response (PR or disease stabilization) in 7 of 10 patients who were assessed. In this group of patients which authors have labeled as heavily pretreated though on looking at patients profile it appears that most of the patients have received just first line treatment similar responses could have been achieved with either single agent cisplatin, $\mathrm{DDP}+5 \mathrm{FU}$ or even with methotrexate. Hong et al in eighties have reported 3 months of response duration and 6.4 months of overall survival OS with cisplatin and 6.1 months with methotrexate. ${ }^{4}$ The median progression-free survival and overall survival of 3.7 months and 5.3 months are not beyond expectation. Cohen et al have reported time to progression of 3.4 months and of OS 8.1 months in similar patients in a phase II study using gefitinib. ${ }^{5}$

Though it is welcome to try a new treatment in quest for better survival and quality of life it may be concluded that time is not yet ripe for routine use of gefitinib outside a clinical trial. Certainly more trials are required to confirm usefulness of small molecules and results from ongoing trials will be more confirmatory one way or other.

\section{REFERENCES:}

1. Bonner JA, Harari PM, Giralt J et al. Radiotherapy plus cetuximab for squamous-cell carcinoma of the head and neck. N Engl J Med.;354 (6):567-78.

2. J. Jansen, J. B. Vermorken, D. Boote, F. Joulain, A. Parthan. Cost-effectiveness analysis of the EORTC 24971 (TAX 323) trial comparing docetaxel plus cisplatin and 5-fluorouracil versus standard treatment (cisplatin and 5-fluorouracil) as induction chemotherapy followed by radiation therapy in locally advanced unresectable squamous cell carcinoma of the head and neck (SCCHN). $J$ Clin Oncol, 2007 (ASCO Proceedings Part I. 2007: Vol 25, No. 18S: 6090 .

3. Rao $R$, Anil $K$, Bansal L et al. Survival benefit and efficacy of gefitinib in recurrent metastatic head and neck cancer. Ind J. Med \& Paed Oncol 2007:28(3):5-10.

4. Hong WK, Schaefer S, Issell B et al. A prospective randomized trial of methotrexate versus cisplatin in the treatment of recurrent squamous cell carcinoma of the head and neck. Cancer. 1983;52(2):206-10

5. Cohen EEW, Rosen F, Stadler WM, et al. Phase II trial of ZD1839 in recurrent or metastatic squamous cell carcinoma of the head and neck. J Clin Oncol 2003;21:19801987.

Atul Sharma

Department of Medical Oncology All India Institute of Medical Sciences

New Delhi 110029

Email:atul1@hotmail.com 